\title{
Internal field study of 21700 battery based on long-life embedded wireless temperature sensor
}

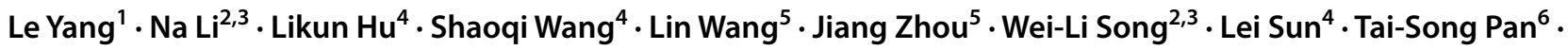 \\ Hao-Sen Chen $^{2,3} \cdot$ Daining Fang ${ }^{2,3}$
}

Received: 14 April 2021 / Accepted: 11 May 2021 / Published online: 22 June 2021

(c) The Author(s) 2021

\begin{abstract}
The safety of lithium-ion batteries is an essential concern where instant and accurate temperature sensing is critical. It is generally desired to put sensors inside batteries for instant sensing. However, the transmission of internal measurement outside batteries without interfering their normal state is a non-trivial task due to the harsh electrochemical environment, the particular packaging structures and the intrinsic electromagnetic shielding problems of batteries. In this work, a novel in-situ temperature sensing framework is proposed by incorporating temperature sensors with a novel signal transmission solution. The signal transmission solution uses a self-designed integrated-circuit which modulates the internal measurements outside battery via its positive pole without package breaking. Extensive experimental results validate the noninterference properties of the proposed framework. Our proposed in-situ temperature measurement by the self-designed signal modulation solution has a promising potential for in-situ battery health monitoring and thus promoting the development of smart batteries.
\end{abstract}

Keywords Internal field study $\cdot$ Embedded sensor $\cdot$ Wireless temperature transmission $\cdot$ Lithium-ion battery

\section{Introduction}

Lithium-ion batteries (LIBs) played an important role in energy strategies from fossil fuels to renewable energy [1,2]. Owing to higher energy density, high nominal voltage, long

Executive Editor: Yuejie Wei

Hao-Sen Chen

chenhs@bit.edu.cn

1 AML, CNMM, Department of Engineering Mechanics, Tsinghua University, Beijing 100084, China

2 Institute of Advanced Structure Technology, Beijing Institute of Technology, Beijing 100081, China

3 Beijing Key Laboratory of Lightweight Multi-Functional Composite Materials and Structures, Beijing Institute of Technology, Beijing 100081, China

4 School of Information and Electronics, Beijing Institute of Technology Beijing, Beijing 100081, China

5 Lishen Research Institute, Tianjin Lishen Battery Joint-Stock Co., Ltd., Tianjin 300384, China

6 State Key Laboratory of Electronic Thin Films and Integrated Devices, University of Electronic Science and Technology of China, Chengdu 610054, China service life and low cost, LIBs have been widely applied in communication technologies, aerospace and electric vehicles [3]. However, due to self-ignite and explosive events with sharply raised temperature over past decade, the safety of LIBs is an essential concern which need to demand the markets $[4,5]$. Therefore, instant and accurate internal information measuring of LIBs is very important.

The complex internal multi-field coupling behaviours during the electrochemical cycles would significantly influence the state of health $(\mathrm{SOH})$ of the LIBs [6-9]. It is essential to analyse and monitor the $\mathrm{SOH}$ of the LIBs to obtain internal information, especially temperature and strain [10-12]. Recently, the BATTERY 2030 + proposed the smart batteries concept, which is based on newly embedded sensors for monitoring status changes in real time. Currently, the temperature of the LIBs has been measured by using different methods, such as thermocouple [13-16], thermo-resistive [17-21], infrared camera [22-27], and optical fibre [28-32]. It is a critical challenge to obtain internal measurement that harsh electrochemical environment, the particular packaging structures and the excellent performance of the LIBs. Only a few methods have been applied to measure internal temperature of the pouch and cylindrical cells [16-18, 31, 32]. For the thermocouple [16] and thermo-resistive [17, 18], the soft 
thin-film sensors with good chemical and thermal stability were preferred for avoiding physical damage to the electrodes and separators. The optical fibre was used to measure the internal temperature of 18,650 battery that placed in the centre of jelly roll $[31,32]$. But optical fibre was too harsh for the thin electrodes, causing damage on electrodes [33]. During the charging and discharging processes, the site of optical fibre has shifted resulting inaccurate temperature measurement that caused by electrode deformation and optical fibres have an impact on the performance of the battery. In order to decrease impact, thin film sensors were located between the electrode and aluminium laminated films that the ionic flow would not be affected between electrodes, which could not monitor the actual internal temperature. On the other hand, the thin film sensors were attached on the electrodes resulted in the electrochemical problem, such as plating [17]. So, electrochemical stability and accurate temperature measurement are need to be urgently solved. Recently, a novel embedded method was developed by Chen et al. [18] that a small region of the active materials on the positive was removed for placing the thin film sensor. The configuration can weaken effect of the sensor on the capacity loss and damage of the active materials. The long-term cyclic experimental results show that the cell with inserting thin film sensor could remain a good cycling performance compared with the normal cell. Up to now, the internal thermal information measurement of the commercial LIBs has been carried out successfully. The conventional wired transmitted way is adopted, while the cable destroys the sealing of the packaging structures, especially for the cylindrical cells. However, it is nontrivial to transmit the internal measurement outside LIBs due to the particular packaging structures of LIBs. The location between the top cap and the steel can be cut to put the sensor into the jelly roll and make the signal transmitter by the cable [31, 32]. However, the transmission of internal measurement outside batteries without interfering their normal state is a non-trivial task due to the harsh electrochemical environment, the particular packaging structures and the intrinsic electromagnetic shielding problems of batteries.

In this paper, in-situ internal temperature field was measured by a novel and facile method based on temperature sensor and wireless transmission, which overcome signal transmission barrier through shell and instability. The wireless transmission was firstly used in internal field measurement of battery. The signal transmission was used by a self-designed integrated-circuit which modulates the internal measurements outside battery via its positive pole without package breaking. This method has no evident impact on battery, especially electrochemistry performance. The stability of battery and wireless sensor were verified by a series of tests, including electrochemical impedance spectroscopy (EIS), charge-discharge cycle, and in-situ internal temperature field under different states. In-situ wireless sensors have provided a new strategy for battery manufacturers for existing technology as well as facilitating future innovation in design and management, which can offer thermal runaway warning for battery safety.

\section{Experiment}

\subsection{Wireless transmission design and manufacturing}

A novel wireless transmission is urgent to be developed for measuring and transmit temperature signal. So, selfdesigned chips were used for wireless signal transmission based on electromagnetic signal transmission by chip carrier. The temperature sensing and transmitting circuit consists of three parts in Fig. 1, temperature sensors as Part (a), data transmission as Part (b) and signal recovery as Part (c). Temperature sensors played role of temperature sensing where a Wheatstone bridge circuit is adopted to connect to the temperature sensor. Once there is a temperature variance, the temperature sensor generates a certain resistance variance which is captured by the Wheatstone bridge circuit. The sensors have been used and calibrated in our previous work [27]. During the calibrate process, the sensors are put in a standard thermostat (DGBELL-BTT-150B) which possess $500 \mathrm{~mm} \times 500 \mathrm{~mm} \times 600 \mathrm{~mm}$ inner space, and could realize precise temperature control. Then, the resistances of the sensors are collected by the resistance test instrument (HPS5132), and the resistance-temperature relationship of the sensor are established. Subsequently, the analog-to-digit (ADC) chip converts the analogy Wheatstone variance to digits. These digits are to be transmitted to the outside of power cell via data transmission. This is a hard but the key part of the temperature sensing and transmitting circuit. Due to limited space of circuits, a full function of signal transmitting circuit, whose mechanical size could be larger than that of a power cell, cannot be mounted inside the power cell.

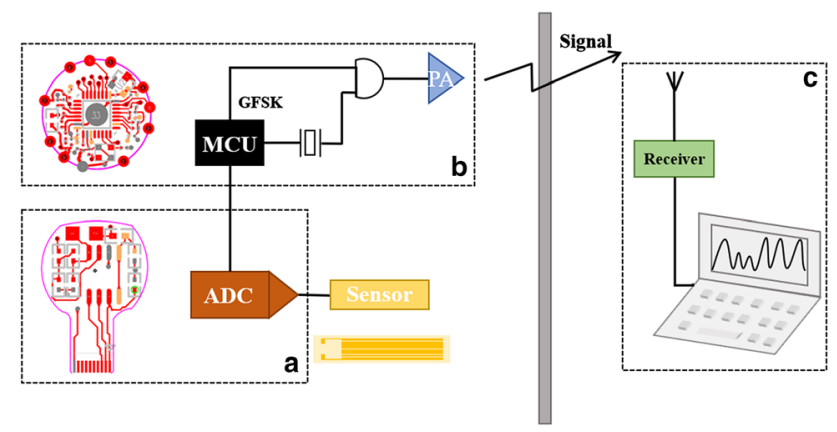

Fig. 1 Schematic illustration of wireless signal transmission. a Temperature sensing, $\mathbf{b}$ data transmitting and $\mathbf{c}$ signal recovery 
Hence, a very simple signal transmitting circuit is designed for our application. For the current in-situ system, the accuracy of the sensor is $0.05{ }^{\circ} \mathrm{C}$, the space between two sensing point on the sensor is $5 \mathrm{~mm}$ and the collection frequency of the resistance tester is $4 \mathrm{~Hz}$. However, the resolution and accuracy of the system could be improved by using more accurate collect instruments.

It is obvious that the modulated signal is very weak due to the fact that there is a large attenuation via the positive pole of power cell. That is, a receiver is expected to recover the temperature information via the weak signal-to-noise ratio (SNR) signal. In signal recovery, the modulated signal is captured via the receiver antenna, and a signal recovery algorithm is designed to recover the temperature variance via the modulated signal. However, compared to the task of data transmitting circuit inside the power cell, the receiver designation is not a hard one because there is no limitation of size or power consumption outside power cell. In short, based on the temperature sensing in Part (a), data transmission in Part (b) and data recovery in Part (c), a temperature sensing system is implemented to capture inner temperature variance inside a power cell. The wireless transmission technology provides an alternative method to solve transmitted problem and was used to transmit internal signal to computer.

\subsection{Integrated wireless sensor into cylindrical battery}

Figure 2 showed schematic diagram of the 21700 lithiumion battery (21700 LIB) with inserted wireless sensor into the jelly roll. A small region of the positive active mate-rials, about $320 \mathrm{~mm}^{2}(8 \mathrm{~mm} \times 40 \mathrm{~mm})$, was removed by N-Methyl pyrrolidone (NMP) to expose $\mathrm{Al}$ foil to integrated thin-film sensor. Thin-film temperature sensor tied chip, width of $5 \mathrm{~mm}$, was put on the surface of the Al current collector. Then positive electrode with wireless sensor, negative electrode and separator were winded together to form cylinder and put into steel shell. The 21700 LIB was assembled and injected electrolyte in the argon-filled glovebox with $\mathrm{H}_{2} \mathrm{O}$ and $\mathrm{O}_{2}$ concentrations $<0.1 \mathrm{ppm}$. The electrolyte was $1 \mathrm{M}$

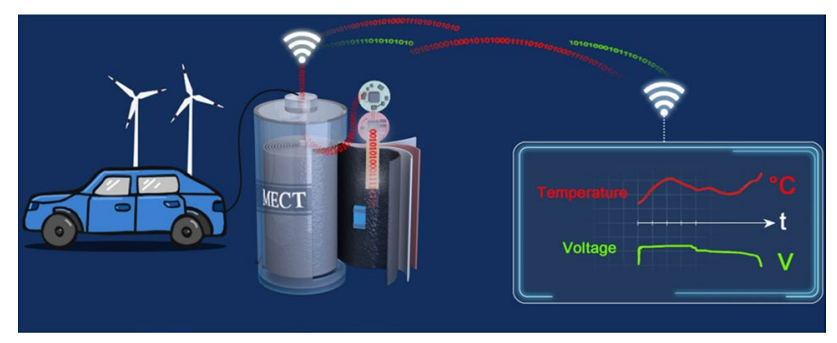

Fig. 2 Schematic of 21700 lithium-ion battery with embedded wireless senor in the core of the cell
$\mathrm{LiPF}_{6}$ with the volume ratio of $\mathrm{EC}: \mathrm{DMC}=1: 1$. And, a 21700

LIB without integrated wireless sensor were also fabricated with above steps. The wireless sensor was composed of thinfilm temperature sensor, chip and demo board, realized stable signal transmission, as shown in Fig. S1.

\subsection{Measurement method}

All the 21700 LIBs, which $\mathrm{LiFePO}_{4}$ and graphite were used as positive and negative electrode, were tested with the voltage between 2.8 and $3.4 \mathrm{~V}$ by the battery testing system of Neware instruments. The electrochemical impedance spectroscopy (EIS) measurements were tested by a BioLogic VMP3 instruments with frequency range from $102 \mathrm{kHz}$ to $0.01 \mathrm{~Hz}$. The internal temperature was measured by wireless sensor and surface temperature was tested simultaneously by thin film sensor. All the temperature measurement and charge-discharge process were executed at the same time. In-situ temperature measurement with thin film sensor was connected with the resistance acquisition system by a printed circuit board (PCB), recorded resistance value.

\section{Results and discussion}

\subsection{Effect of wireless sensor on the battery}

The X-ray tomography images of sensor in cylindrical cells were shown in Fig. S2, which displayed the location of sensor and chips. In order to demonstrate electrochemical stability of 21700 LIBs with wireless sensor, the electrochemical performances were tested by Neware instrument and electrochemical workstation. The batteries with wireless sensor were named integrated batteries and normal batteries without wireless sensor. As shown in Fig. 3, integrated batteries have exhibited similarly capacity at the current density of $1500 \mathrm{~mA}$ compared to normal batteries without wireless sensor. The discharge capacity of integrated batteries has shown a slightly attenuation at the first cycle in Fig. 3a due to remove a small area of the active materials to embed sensors, reduced form 1.19 Ah to $1.16 \mathrm{Ah}$. The capacity of integrated batteries was stable during 100 cycles, which verified that the wireless sensor had no evident impact on battery. In addition, normal and integrated batteries were cycled at the current density of 1.5 A. From the Fig. 3c, the discharge capacity of the normal batteries dec creased form $1.19 \mathrm{Ah}$ to $1.15 \mathrm{Ah}$, which were $3.3 \%$ capacity reduction. But the integrated batteries have kept relative capacity and good stable cycle performance, contain at $1.16 \mathrm{Ah}$. The coulombic efficiency was close to $99.9 \%$. The above results demonstrated that integrated wireless sensor has exhibited almost no effect on the capacity and cycle performance. To evaluate the effects of integrated wireless sensor of LIBs, the 

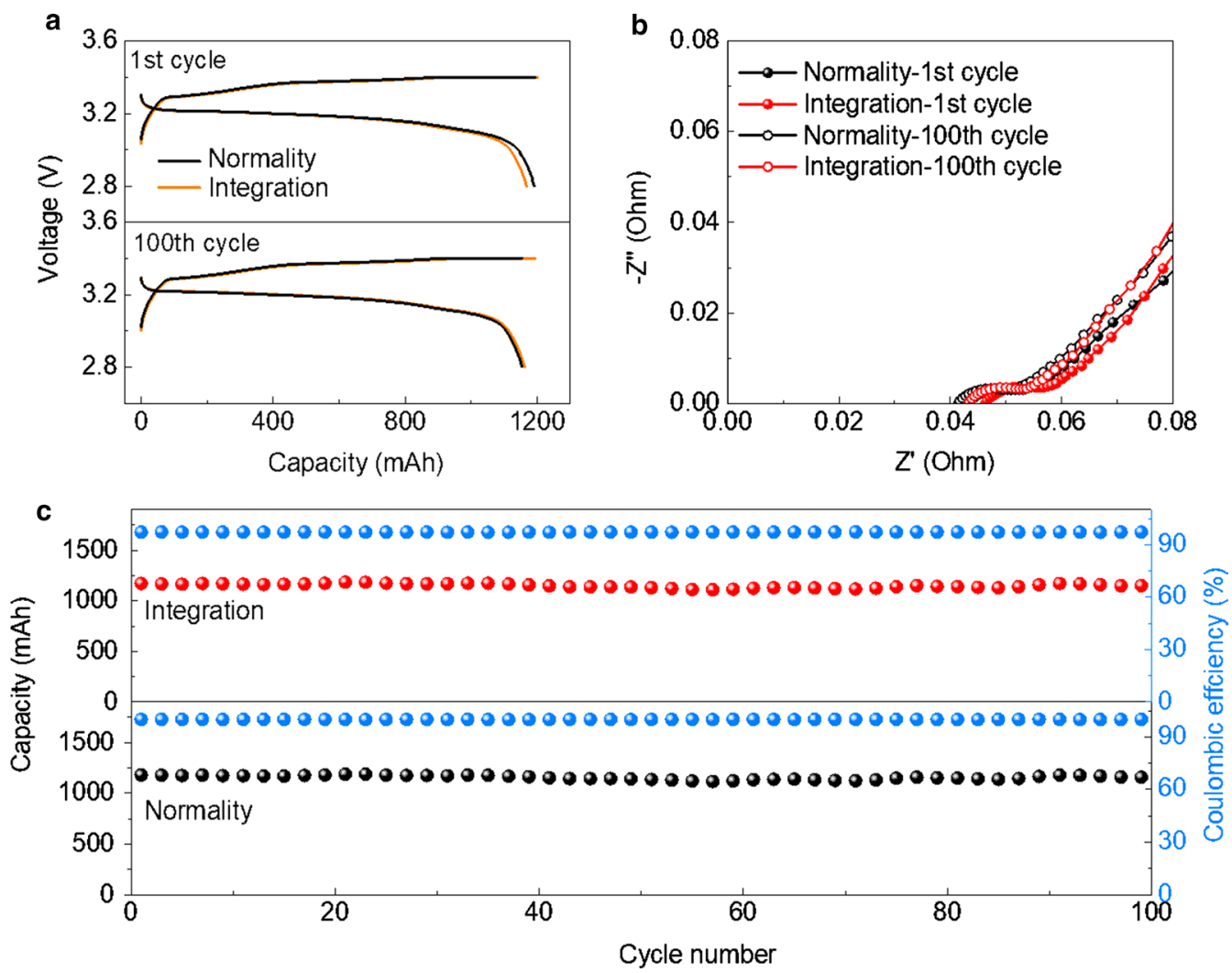

Fig. 3 Electrochemical performance of 21700 lithium-ion battery with or without wireless sensor. a The charge-discharge curves. b The electrochemical impedance spectra. $\mathbf{c}$ The charge-discharge cycles and coulombic efficiency at the current density of $1500 \mathrm{~mA}$

normal and integrated batteries were analysed under different cycles by the electrochemical impedance spectroscopy (EIS). The Nyquist curves of normal and integrated batteries with 1 st and 100th cycle were displayed in Fig. 3b. Intersection point with $Z$ ' axis represents internal resistance which mainly related to ohmic resistance of electrolyte and internal resistance of the electrodes. Form the Fig. 3b, intersection point shifted a slightly due to little changes in the contact between the electrodes which may be caused by integrated wireless sensor into cathode. The semicircles were related to the solid electrolyte interface and resistance of charge transport charge transport. In the low frequency, it corresponded to the Li-ion diffusion in the solid electrodes. The EIS data of the normal and integrated battery became slightly larger due to electrode structure changed with cycling. And the behaviours in each frequency with normal and integrated batteries had similar changes that demonstrated the integrated wireless sensor has no significant impact on battery. Considering cycle performance and EIS, it can be verified that this novel method, integrated wireless sensor, had little impact on the electrochemical performance of batteries.

\subsection{The temperature measurements of the battery}

For the purpose of measuring the temperature changes of integrated batteries, the internal temperature and surface temperature were tested by wireless sensor and surface sensor on battery shell, respectively. As shown in Fig. 4, the measured temperature changes, internal and surface temperature, were tightly consistent with charged and discharged process. It is noted that the response and transmission time of the wireless sensor were sufficient to receive the internal temperature. Owing to the hostile environment of the battery, the stability of wireless sensor is very important, which can offer in-situ temperature measurement stably under a long-term cycle. From the Fig. 4a, the internal temperatures of 1 st cycle and 100th cycle were measured at the constant current of $1500 \mathrm{~mA}$. Even though no difference was observed between first cycle and 100th cycle, it is clearly stated that the wireless sensor can maintain the stable performance under the long-cycle condition in the corrosive environment of battery. So, it is certified that the corrosive environment of battery has no effect on the wireless sensor.

Furthermore, the temperature of 21700 LIBs changed with charge-discharge rate. As shown in Fig. 4b, the internal and surface temperature increased as the result of heat 

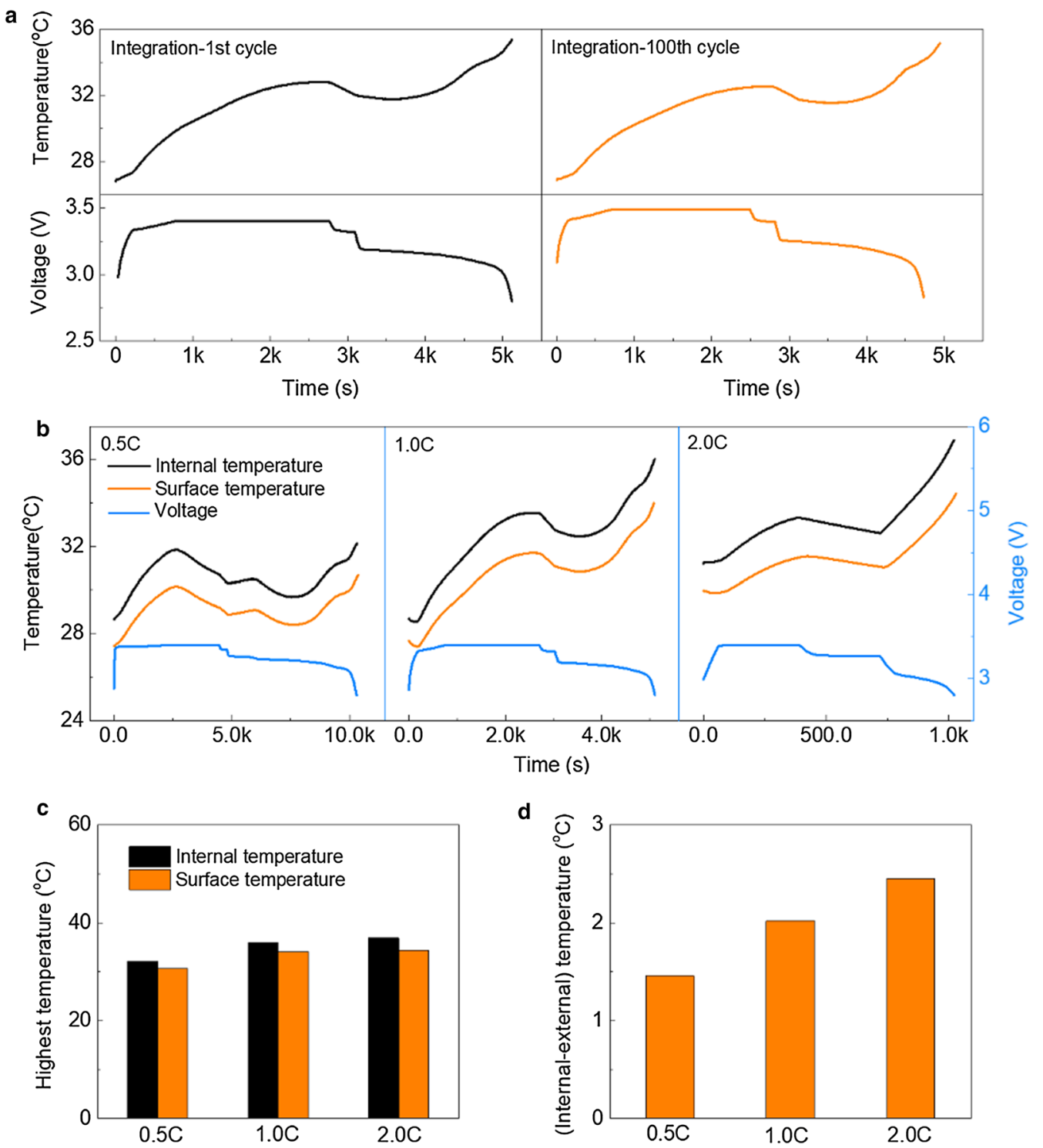

Fig. 4 Temperature measurements. a The internal and surface temperature between the 1st cycle and 100th cycle. b The internal and surface temperature distribution with different rates. $\mathbf{c}$ The highest temperature in internal and surface of integrated battery. $\mathbf{d}$ The average temperature difference between internal and surface of integrated battery

generate during charge-discharge processes with different current density of $0.5 \mathrm{C}, 1 \mathrm{C}$ and $2 \mathrm{C}$. Neither internal nor surface temperature increased continually with the increase of $\mathrm{C}$ rates. In Fig. 4b, c, the maximum temperature rise can reach about $8{ }^{\circ} \mathrm{C}$ and the maximum internal temperature was nearly $36^{\circ} \mathrm{C}$. Even though no lag was observed between the internal and surface temperature, it is shown that in all rates the internal temperature is significantly higher than surface temperature. The average temperature difference between the internal and surface in Fig. 4d were caused by the thin film sensor sites and small radial thermal conductivity of cylindrical battery. And in all cases presented the temperature of discharge process is significantly higher than that of charge process. But the internal temperature would decrease during the charge process. In order to simulate the real work conditions, the battery was tested under a nonadiabatic condition, thus the heat generated was not enough to make the temperature rise due to the heat dissipation during the constant voltage charge process. These results show the typical non-equilibrium thermal process inner the LIBs during the electrochemical cycles. The temperature is various with the position changes along the radial direction of the 21700 LIBs, the heat exchange of the battery with the environment could also influence the temperature field evolution. However, the wireless sensor can measure accurately the internal temperature and transmit stable signals, 


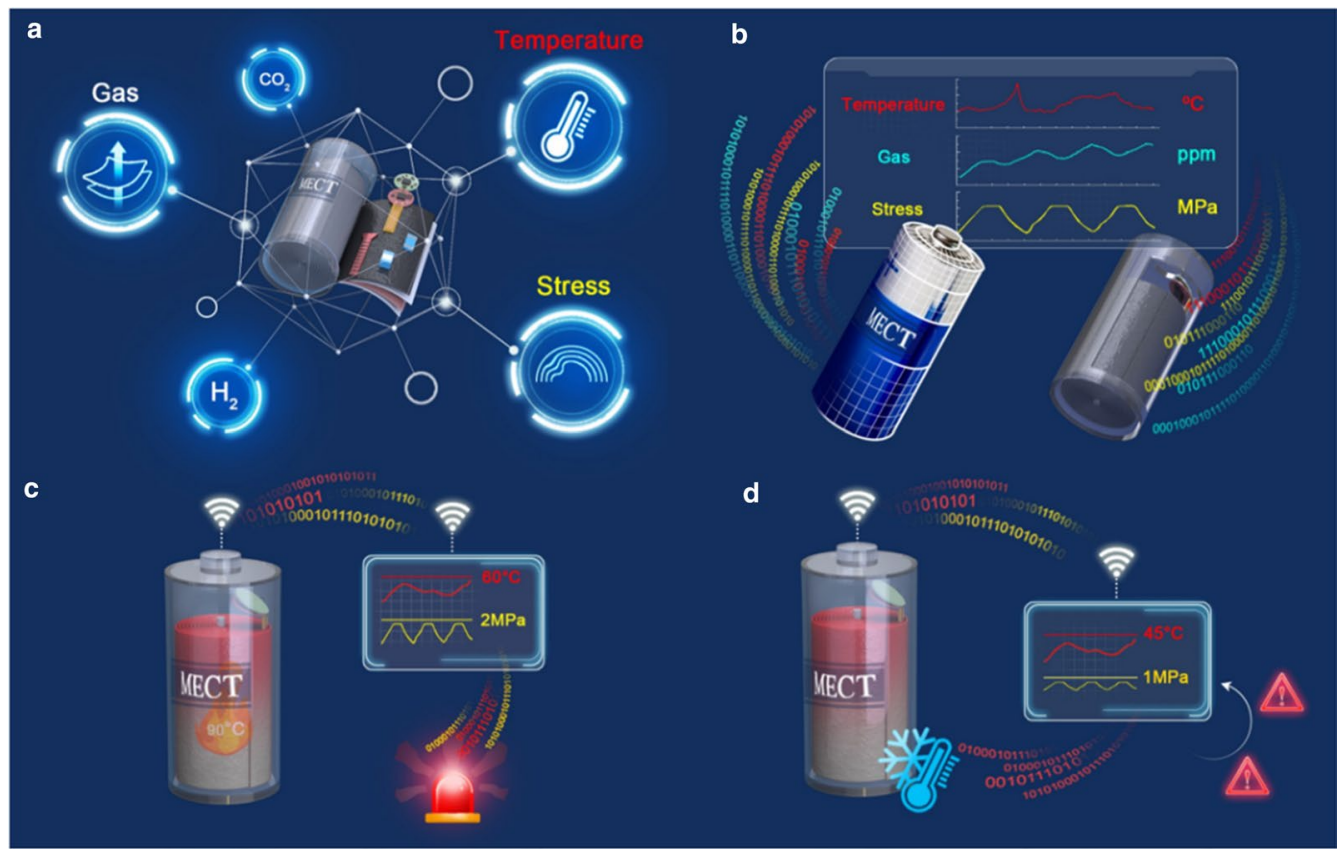

Fig. 5 Propescts. a In-situ monitor internal information of battery. b Digtal twin of battery with all information. $\mathbf{c}$ The early warning of battery thermal runaway. $\mathbf{d}$ Intelligent management (self-healing) of battery

and the sensors could inserted at the difference locations along the radial direction of the battery, and could be used to in-situ study the non-equilibrium thermal process. Consequently, this method, integrated wireless sensor, provides a new perspective to manufacturers to improve battery design, such as safety and performance improvement. Consequently, this method, integrated wireless sensor, provides a new perspective to manufacturers to improve battery design, such as safety and performance improvement.

\section{Conclusions}

In summary, a novel in-situ method by integrated wireless sensor that signal transmission was used by a self-designed integrated-circuit, was proposed to measure internal temperature of battery. The integrated wireless sensor has almost no effect on the electrochemical performance, which was demostrated by EIS and charge-dicharge cycle. The wireless sensor can measure accuratly the internal temperature and transmit stable signal in real-time with different current rates. The significant divergence between internal and surface of battery temperatures was caused by small radial thermal conductivity of cylindrical battery, which demonstated the internal temperature can better reflect the state of health of the battery. So this in-situ measurement by wireless sensor has the potential to drive improvement in battery management systems to maintain excellent performance and understand state of health during deployment in actual scenarios.

Furthermore, we can develop this wireless sensor method to measure all internal information that use to monitor battery status in realtime. When different kinds of sensors are integrated into multiple sites of battery in Fig. 5a, temperature, stress and gas signal will been obtained in the same time. In-situ real-time monitoring through smart sensors is invaluable to researchers and engineers, which is attribute develop an intelligent responsive battery management system. The smart sensors and intelligent management system can promote the realization of digital twins in Fig. 5b. The wireless sensor technology will be used to realize the early warning of battery thermal runaway, as shown in Fig. 5c. The wireless sensor technology is consistent with concept of smart battery and smart management, which can inspired by the medical science by developing self-healing to improve battery lifetime and safety in practice in Fig. 5d. The wireless sensor technology that combined with artificial intelligence, robotics and smart system, would contribute to achieve ultrahigh-performance, safe and affordable batteries. In a word, it is foreseen that the wireless sensing technology can be extended to monitor all internal filed signal, intelligently battery management and digital twin.

Supplementary Information The online version contains supplementary material available at https://doi.org/10.1007/s10409-021-01103-0. 
Acknowledgements This work was supported by the Beijing Municipal Science and Technology Commission (Grant Z191100002719007) and the National Natural Science Foundation of China (Grant 11672341).

Open Access This article is licensed under a Creative Commons Attribution 4.0 International License, which permits use, sharing, adaptation, distribution and reproduction in any medium or format, as long as you give appropriate credit to the original author(s) and the source, provide a link to the Creative Commons licence, and indicate if changes were made. The images or other third party material in this article are included in the article's Creative Commons licence, unless indicated otherwise in a credit line to the material. If material is not included in the article's Creative Commons licence and your intended use is not permitted by statutory regulation or exceeds the permitted use, you will need to obtain permission directly from the copyright holder. To view a copy of this licence, visit http://creativecommons.org/licenses/by/4.0/.

\section{References}

1. Winter, M., Barnett, B., Xu, K.: Before Li ion batteries. Chem. Rev. 118, 11433-11456 (2018)

2. Liu, X., Ren, D., Hsu, H., et al.: Thermal runaway of lithium-ion batteries without internal short circuit. Joule 2, 2047-2064 (2018)

3. Liu, D., Shadike, Z., Lin, R., et al.: Review of recent development of in situ/operando characterization techniques for lithium battery research. Adv. Mater. 31, 1806620 (2019)

4. Feng, X., Ouyang, M., Liu, X., et al.: Thermal runaway mechanism of lithium-ion battery for electric vehicles: a review. Energy Storage Mater. 10, 246-267 (2018)

5. Han, X., Lu, L., Zheng, Y., et al.: A review on the key issues of the lithium-ion battery degradation among the whole life cycle. Etransportation 1, 100005 (2019)

6. Ji, L., Guo, Z.: Analytical modeling and simulation of porous electrodes: Li-ion distribution and diffusion-induced stress. Acta. Mech. Sin. 34, 187-198 (2018)

7. Xie, H., Kang, Y., Song, H., et al.: In situ method for stress measurements in film-substrate electrodes during electrochemical processes: key role of softening and stiffening. Acta. Mech. Sin. 36, 1319-1335 (2020)

8. Zhang, Y., Guo, Z.: Numerical computation of central crack growth in an active particle of electrodes influenced by multiple factors. Acta. Mech. Sin. 34, 706-715 (2018)

9. Liu, M., Lu, B., Shi, D., et al.: Two-dimensional analysis of progressive delamination in thin film electrodes. Acta. Mech. Sin. 34, 359-370 (2018)

10. Raijmakers, L.H.J., Danilov, D.L., Eichel, R.A., et al.: A review on various temperature-indication methods for $\mathrm{Li}$-ion batteries. Appl. Energy 240, 918-945 (2019)

11. Cheng, X., Pecht, M.: In situ stress measurement techniques on Li-ion battery electrodes: a review. Energies 10, 591 (2017)

12. Feng, X., Yang, L., Zhang, M., et al.: Failure mechanics inner lithium-ion batteries: in-situ multi-field experimental methods. Energy Storage Sci. Technol. 8, 1062-1075 (2019)

13. Chalise, D., Shah, K., Halama, T., et al.: An experimentally validated method for temperature prediction during cyclic operation of a Li-ion cell. Int. J. Heat Mass Transf. 112, 89-96 (2017)

14. Grandjean, T., Barai, A., Hosseinzadeh, E., et al.: Large format lithium ion pouch cell full thermal characterisation for improved electric vehicle thermal management. J. Power Sources 359, 215225 (2019)

15. Drake, S.J., Martin, M., Wetz, D.A., et al.: Heat generation rate measurement in a Li-ion cell at large C-rates through temperature and heat flux measurements. J. Power Sources 285, 266-273 (2015)

16. Mutyala, M.S.K., Zhao, J., Li, J., et al.: In-situ temperature measurement in lithium ion battery by transferable flexible thin film thermocouples. J. Power Sources 260, 43-49 (2014)

17. Fleming, J., Amietszajew, T., Charmet, J., et al.: The design and impact of in-situ and operando thermal sensing for smart energy storage. J. Energy Storage 22, 36-43 (2019)

18. Zhu, S., Han, J., An, H.Y., et al.: A novel embedded method for in-situ measuring internal multi-point temperatures of lithium-ion batteries. J. Power Sources 456, 227981 (2020)

19. Lee, C.Y., Lee, S.J., Hung, Y.M., et al.: Integrated microsensor for real-time microscopic monitoring of local temperature voltage and current inside lithium-ion battery. Sens. Actuator A 253, 59-68 (2017)

20. Lee, C.Y., Chuang, S.M., Lee, S.J., et al.: Flexible micro sensor for in-situ monitoring temperature and voltage of coin cells. Sens. Actuator A 232, 214-222 (2015)

21. Lee, C.Y., Weng, F.B., Huang, Y.P., et al.: Real-time monitoring of internal temperature and voltage of high-temperature fuel cell stack. Electrochim. Acta 161, 413-419 (2015)

22. Bolsinger, C., Birke, K.P.: Effect of different cooling configurations on thermal gradients inside cylindrical battery cells. J. Energy Storage 21, 222-230 (2019)

23. Waldmann, T., Bisle, G., Hogg, B.I., et al.: Influence of cell design on temperatures and temperature gradients in lithium-ion cells: an in operando study. J. Electrochem. Soc. 162, A921 (2015)

24. Anthony, D., Wong, D., Wetz, D., et al.: Non-invasive measurement of internal temperature of a cylindrical Li-ion cell during high-rate discharge. Int. J. Heat Mass Transf. 111, 223-231 (2017)

25. Zhu, S., Han, J., Pan, T.S., et al.: A novel designed visualized $\mathrm{Li}$-ion battery for in-situ measuring the variation of internal temperature. Extreme Mech. Lett. 37, 100707 (2020)

26. Zhu, S., Han, J., Wang, Y., et al.: In-situ heat generation measurement of the anode and cathode in a single-layer lithium-ion battery cell. Int. J. Energ. Res. 44, 9141-9148 (2020)

27. Du, X., Wu, Q., Wang, Y.N., et al.: Visualizing two-dimensional internal temperature distribution in cylindrical Li-ion cells. J. Power Sources 446, 227343 (2020)

28. Nascimento, M., Novais, S., Ding, M.S., et al.: Internal strain and temperature discrimination with optical fiber hybrid sensors in Li-ion batteries. J. Power Sources 410-411, 1-9 (2019)

29. Nascimento, M., Ferreira, M.S., Pinto, J.L.: Real time thermal monitoring of lithium batteries with fiber sensors and thermocouples: A comparative study. Measurement 111, 260-263 (2017)

30. Novais, S., Nascimento, M., Grande, L., et al.: Internal and external temperature monitoring of a Li-ion battery with fiber Bragg grating sensors. Sensors 16, 1394-1399 (2016)

31. Fleming, J., Amietszajew, T., McTurk, E., et al.: Development and evaluation of in-situ instrumentation for cylindrical Li-ion cells using fibre optic sensors. HardwareX 3, 100-109 (2018)

32. Somerville, L., Ferrari, S., Lain, M.J., et al.: An in-situ reference electrode insertion method for commercial 18650-type cells. Batteries 4, 18 (2018)

33. Martiny, N., Mühlbauer, T., Steinhorst, S., et al.: Digital data trans-mission system with capacitive coupling for in-situ temperature sensing in lithium-ion cells. J. Energy Storage 4, 128-134 (2015)

Publisher's Note Springer Nature remains neutral with regard to jurisdictional claims in published maps and institutional affiliations. 